\title{
Correction: Impact of seaweed intake on health
}

Utako Murai - Kazumasa Yamagishi (i) - Rie Kishida - Hiroyasu Iso

Published online: 15 October 2020

(c) The Author(s), under exclusive licence to Springer Nature Limited 2020

Correction to: European Journal of Clinical Nutrition https://doi.org/10.1038/s41430-020-00739-8.
Since publication of the article the authors noticed that " 2 . Ischemic heart disease" of Fig. 1 was missing. This has now been corrected in the HTML and PDF versions of the paper. 